\title{
Opinion
}

\section{Definition of K-BTE medical laser device}

\author{
Nick Kostovic* \\ Bio Technological Health Center, Inc., 3838 Carson Street, Suite 307, Torrance, CA 90503, Los Angeles, USA
}

The K-BTE device releases electrons, enriched with natural acids, and is capable of "melting by burning off," then dispersing, any number of benign or malignant tumors with metastasis in the brain or any other physical organ-with no harm to healthy cells.

It "Melts by burning off" then disperses many different types of damaged, diseased or necrotic cells in the brain-stopping or reversing any number of neurological disorders, with no harm to the healthy cells.

It "Melts by burning off" then disperses dead cells from the fiber of atrophied muscle, bone and cartilage tissue-as well as plaque from the vascular system-with no harm to healthy cells.

The K-BTE device attracts and transfers light-which consists of elemental bio chemical charges and electron neutrinos - into the brain. This fosters the regeneration, recovery and re-growth of neurons in the brain, thereby regaining its ability to send impulses throughout the body while improving mobility.

\section{The K-BTE device is intended to be used in}

Melting and destroying malignant cancer cells in the brain - or any other physical organ - accomplished with no side effects and no harm to healthy cells.

Melting cleanses internal plaque from the vascular system in the brain or any other physical organ. This will prevent strokes, heart attacks, diabetes and many different neurological disorders.

Melting cleanses, the fiber of dead cells - dispersing them and making space for the formation of new and healthy cells. This is the most important step for a vital, longer lasting life. It can also be used for cosmetic and aesthetic care since we are able to tighten the skin on the face, neck, arms and legs.

When the K-BTE device releases enriched electrons combined with natural acids, including amino acids, this "bioelectricity" stimulates the release of new cells. These new cells are called daughter cells - known as the "Mitosis phase" in cell growth. In addition, the enriched electrons, in this "bioelectricity," stimulate the growth of new cells, allowing them to split. This is known as the "Mitotic phase". The result is the rebuilding of the fiber of muscle, bone and cartilage in any physical organ.

The K-BTE device has the capability to cleanse the body of radioactive radiation particles as well as different types of toxic biochemical nerve gas.

The K-BTE device also has the ability of maintaining the bone and muscle tissue of astronauts in space. Currently, Astronauts' orbits are limited to six (6) months since they suffer from deterioration of bone tissue and show significant muscle atrophy. With that said, there are no circumstances - such as gravitation, vacuum, or any type of energetic field irradiation - that would have any detrimental influence on K-BTE's own energetic field. This would allow for the maintenance of Astronauts' bone and muscle tissue while in space thus preventing any decline or deterioration.

This enriched-electrons-radiation-emitting-process is absolutely non-radioactive, non-toxic and is, in no way, "shock therapy."
Copyright: (C2016 Kostovic N. This is an open-access article distributed under the terms of the Creative Commons Attribution License, which permits unrestricted use, distribution, and reproduction in any medium, provided the original author and source are credited.
Correspondence to: Nick Kostovic, Bio Technological Health Center, Inc., 3838 Carson Street, Suite 307, Torrance, CA 90503, Los Angeles, USA, Tel: +1-310-543-0309/+1-310-381-9105; Fax: +1-310-540-6946; E-mail: nick@ kbhealthcenter.com

Received: October 05, 2016; Accepted: October 20, 2016; Published: October 24, 2016 